\title{
Effect of legume and perennial ryegrass herbages on in vitro methane output using the total gas production technique
}

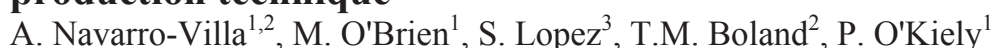

${ }^{1}$ Teagasc, Grange Beef Research Centre, Dunsany, Co. Meath, Ireland

${ }^{2}$ School of Agriculture, Food Science \& Veterinary Medicine, University College Dublin, Belfield, Dublin 4, Ireland,

${ }^{3}$ Departamento de Producción Animal, Universidad de León, Leon, Spain

Email: a.navarrovilla@gmail.com

Introduction In grass based beef production systems, the type and quality of herbage offered may have an influence on enteric methane $\left(\mathrm{CH}_{4}\right)$ emissions. Methane not only contributes to global warming (McGettigan el al., 2008), it also represents an energy loss from the diet. The current study aims to compare the methane output from contrasting herbages at various stages of maturity.

Material and methods Herbage samples were obtained from a randomized complete block $(n=4)$ designed field plot experiment in each of two successive years $(\mathrm{Y})$. The herbage treatments $(\mathrm{Hb})$ were Lolium perenne that received $0 \mathrm{~kg}$ [G1] or $150 \mathrm{~kg}$ [G2] of inorganic $\mathrm{N}$ fertiliser/ha/cut and two cultivars of Trifolium pratense (Merviot [L1] and Ruttinova [L2]). Independent plots of each herbage were harvested (Hv) twice during the primary growth (late May [I] \& mid June [II]) and during an autumn regrowth [III]. Dried $\left(40^{\circ} \mathrm{C} ; 48 \mathrm{~h}\right)$ milled $(1 \mathrm{~mm})$ herbage samples were incubated in rumen fluid according to Mauricio et al. (1999). Methane and total VFA concentrations were determined using gas chromatography, for gas and liquid samples taken 24h after inoculation. Data were analysed using the PROC MIXED procedure in SAS considering observation from different years as repeated measures.

Results Chemical and fermentation characteristics of herbages are shown in Table 1. Crude protein (CP) content of the herbages was higher in the autumn (III) than in the early summer harvests (I \& II) and negatively correlated $(\mathrm{P}<0.001)$ with water soluble carbohy-drates (WSC) and NDF content. Both $\mathrm{Hb}$ and Hv significantly influenced $(\mathrm{P}<0.05)$ all variables displayed in Table 1. Red clovers ( $\mathrm{L} 1 \& \mathrm{~L} 2)$ had a lower $\mathrm{CH}_{4}$ output per $\mathrm{g}$ DM incubated $(\mathrm{P}<0.05)$ compared to nonfertilised ryegrass (G1) and fertilised ryegrass (G2) had a lower output of $\mathrm{CH}_{4}$ per g digested $(\mathrm{P}<0.05)$ than L1. Autumn harvest (III) had a lower output of $\mathrm{CH}_{4}$ per $\mathrm{g}$ DM digested $(\mathrm{P}<0.05)$ than harvests I and II. There was a negative correlation $(\mathrm{P}<0.001)$ between $\mathrm{CP}$ content and $\mathrm{CH}_{4}$ output (per g inc. and dig.).

Table 1 Herbage chemical composition, in vitro methane output and fermentation characteristics

\begin{tabular}{|c|c|c|c|c|c|c|c|c|c|}
\hline $\begin{array}{l}\text { Herbage } \\
(\mathrm{Hb})\end{array}$ & $\begin{array}{l}\text { Harvest } \\
(\mathrm{Hv})\end{array}$ & $\begin{array}{l}\text { CP } \\
g / k g D M\end{array}$ & $\begin{array}{l}\text { WSC }{ }^{1} \\
g / k g D M\end{array}$ & $\begin{array}{l}\mathrm{NDF} \\
g / \mathrm{kg} D M\end{array}$ & $\begin{array}{l}\mathrm{aDMd}^{2} \\
\mathrm{~g} / \mathrm{g}\end{array}$ & $\begin{array}{l}\mathrm{CH}_{4} \mathrm{inc}^{3} \\
\mathrm{mmol} / \mathrm{g} \mathrm{DM}\end{array}$ & $\begin{array}{l}\mathrm{CH}_{4} \mathrm{dig}^{4} \\
\mathrm{mmol} / \mathrm{g} \mathrm{DM}\end{array}$ & $\begin{array}{l}\text { tVFA }^{5} \\
\mathrm{mmol} / L^{2}\end{array}$ & $\mathrm{C} 2: \mathrm{C}^{6}$ \\
\hline \multirow[t]{3}{*}{ G1 } & I & 98 & 236 & 475 & 0.65 & 1.82 & 2.78 & 53 & 2.85 \\
\hline & II & 100 & 137 & 542 & 0.58 & 1.65 & 2.83 & 46 & 2.77 \\
\hline & III & 186 & 131 & 474 & 0.67 & 1.66 & 2.50 & 48 & 3.02 \\
\hline \multirow[t]{3}{*}{ G2 } & I & 170 & 97 & 503 & 0.63 & 1.67 & 2.65 & 49 & 3.11 \\
\hline & II & 163 & 41 & 563 & 0.54 & 1.52 & 2.84 & 44 & 3.01 \\
\hline & III & 261 & 86 & 451 & 0.71 & 1.55 & 2.22 & 46 & 3.24 \\
\hline \multirow[t]{3}{*}{ L1 } & I & 175 & 65 & 404 & 0.54 & 1.64 & 3.05 & 45 & 3.64 \\
\hline & II & 176 & 33 & 494 & 0.52 & 1.48 & 2.87 & 41 & 3.36 \\
\hline & III & 284 & 56 & 341 & 0.63 & 1.50 & 2.45 & 43 & 3.40 \\
\hline \multirow[t]{3}{*}{ L2 } & I & 180 & 66 & 421 & 0.58 & 1.62 & 2.68 & 45 & 3.64 \\
\hline & II & 186 & 53 & 439 & 0.49 & 1.50 & 3.06 & 42 & 3.46 \\
\hline & III & 287 & 52 & 354 & 0.63 & 1.50 & 2.37 & 44 & 3.35 \\
\hline SEM $(\mathrm{Hb} \times \mathrm{Hv} \times \mathrm{Y})^{7}$ & & 7.3 & 12.8 & 15.1 & 0.026 & 0.034 & 0.111 & 1.7 & 0.084 \\
\hline $\mathrm{Y}$ & & $<0.001$ & $<0.001$ & $<0.001$ & $<0.001$ & $<0.001$ & 0.329 & $<0.01$ & $<0.001$ \\
\hline $\mathrm{Hb} \times \mathrm{Hv}$ & & 0.309 & $<0.001$ & $<0.01$ & 0.155 & 0.569 & 0.064 & 0.534 & $<0.05$ \\
\hline $\mathrm{Hb} \times \mathrm{Y}$ & & 0.063 & $<0.001$ & $<0.05$ & 0.522 & 0.285 & 0.587 & 0.087 & $<0.05$ \\
\hline Hv x Y & & $<0.001$ & $<0.01$ & $<0.001$ & $<0.001$ & $<0.05$ & $<.001$ & 0.504 & $<0.001$ \\
\hline $\mathrm{Hb} \times \mathrm{Hv} \times \mathrm{Y}$ & & 0.125 & $<0.001$ & 0.052 & $<0.05$ & 0.001 & 0.332 & 0.017 & 0.356 \\
\hline
\end{tabular}

${ }^{1}$ WSC- Water soluble carbohydrates; ${ }^{2}$ aDMd- apparent DM disappearance; ${ }^{3} \mathrm{CH}_{4}$ inc- methane expressed per g of DM incubated; ${ }^{4} \mathrm{CH}_{4}$ dig- methane expressed per g of DM digested; ${ }^{5}$ tVFA-total fermentation VFA; ${ }^{6} \mathrm{C} 2: \mathrm{C} 3$ - ratio acetate to propionate; ${ }^{7} \mathrm{n}=4$.

Conclusions In general, $\mathrm{CH}_{4}$ output was lowest in herbages with higher $\mathrm{CP}$ content. Fertilised ryegrass had a lower $\mathrm{CH}_{4}$ output per g DM digested than Merviot red clover. Autumn harvest showed a lower methanogenic potential than early summer harvests.

Acknowledgements Funding for this research was provided under the National Development Plan, through the Research Stimulus Fund, administered by the Department of Agriculture, Fisheries \& Food, Ireland.

\section{References}

McGettigan, M., Duffy P., Hyde B., and O’Brien P. 2008. Ireland National Inventory Report 2008. Greenhouse Gas Emissions 1990-2006. Environmental Protection Agency, Johnstown Castle Estate, Wexford, Ireland.

Mauricio, R.M., Mould, F.L., Dhanoa, M.S., Owen, E., Channa, K.S. and Theodorou, M.K. 1999. Animal Feed Science and Technology. 79: 321-330. 